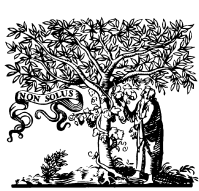

ELSEVIER

\title{
3-D wave propagation in fluid-filled irregular boreholes in elastic formations
}

\author{
António J.B. Tadeu* ${ }^{*}$, Paulo F.A. Santos \\ University of Coimbra, Department of Civil Engineering, 3030 Coimbra, Portugal
}

Accepted 25 February 2001

\begin{abstract}
Different seismic testing techniques rely on the propagation of acoustic waves in fluid-filled boreholes from sources placed within the borehole and in the solid media. The interpretation of the signals recorded relies on understanding how waves propagate in the borehole and its immediate vicinity. It is known that very complex wave patterns can arise, depending on the distance between the source and the receiver, and their placement and orientation relative to the axis of a circular borehole. The problem becomes more complex if the cross-section is not circular, conditions for which analytical solutions are not known. In this work, the Boundary Element Method (BEM) is used to evaluate the three-dimensional wave field elicited by monopole sources in the vicinity of a fluid-filled borehole. This model is used to assess the effects of the receiver position on the propagation of both axisymmetric and non-axisymmetric wave modes when different borehole cross-sections are used. Both frequency vs. axial-wave number responses and time-domain responses are calculated. (C) 2001 Elsevier Science Ltd. All rights reserved.
\end{abstract}

Keywords: Wave propagation; Fluid-filled borehole; Boundary element method; Two-and-a-half-dimensional problem; Dispersion of waves; Synthetic waveforms

\section{Introduction}

A large number of researchers have addressed the problem of wave propagation along fluid-filled boreholes from sources inside and outside the borehole, because of its importance to acoustic logging, vertical profiling and cross-hole surveying.

The problem concerning the interaction of elastic waves with fluid-filled or air-filled cylinders has received considerable attention. Before the 1980s, most of the work published focused on dynamic stresses and wave dispersion characteristics, with very little being done on particle motions at a certain point (documented in an excellent monograph by Pao and Mow [1]).

The propagation of waves along a borehole boundary was studied nearly half a century ago by Biot [2], who derived the dispersion equation for guided waves in a borehole, and used it to find the phase and group velocities for these waves. More recently, a number of investigators have addressed the propagation of waves along fluid-filled boreholes from sources aligned with the borehole axis, because of its importance in

\footnotetext{
* Corresponding author. Tel.: +351-239-797-201; fax: +351-239-797190.

E-mail address: tadeu@dec.uc.pt (A.J.B. Tadeu).
}

acoustic logging. The interaction of elastic plane waves incident upon a fluid or air-filled borehole has been investigated in a context of vertical profiling and cross-hole surveying. Blair [3] considered the case of a P-wave normally incident on the borehole axis, while Schoenberg [4] studied the problem of plane compressional or shear waves impinging on the fluid borehole at arbitrary angles, providing explicit formulas that are valid only for low frequencies. Lovell and Hornby [5] returned to this problem, developing expressions valid for all frequencies and incidence angles.

Numerical methods have also been used to study how wave amplitude and attenuation are influenced by the formation characteristics of the material, by the type of fluid in the borehole, and by the source [6-11]. The inverse problem of estimating the mechanical properties of the formation has also been the object of research [12,13], and this work was later extended to consider the state of fracturing and the presence of damaged zones around the borehole [14-16]. The effects of the formation anisotropy on the fluid-filled borehole wave propagation have been analyzed by different authors. Transversely isotropic formations, with the symmetry axis aligned with the axis of the borehole, and the use of monopole and dipole acoustic logs have also been investigated [17-19]. The perturbation and the finite element methods were also developed to study 
borehole normal modes and waveforms in the anisotropic formation [20-23] derived the radiation patterns of typical downhole seismic sources inside a fluid-filled borehole embedded in a transversely isotropic formation. Anisotropy has also been included in crosswell tomography techniques [24].

Kurkjian et al. [25] proposed a numerical technique for modeling downhole seismic data in crosswell configurations, splitting the problem into three distinct parts: the first takes into account the generation of tube waves in the source well, the second assumes the transmission from the source well using a preexisting code, and at third computes the response at the receivers by applying White's quasistatic approximation. In their method, the boreholes are discretized into small elements. Later, Peng et al. [26] make use of both the borehole coupling theory and the global matrix formulation for calculating synthetic seismograms in a layered medium. This model does not need discretization along the borehole, and the method applies to open, cased and partially filled boreholes.

Randall [27] studied monopole and dipole acoustic logs using a finite difference method, applying a staggered grid in a 2-D cylindrical coordinate. A true 3-D finite difference method, utilizing a parallel computing scheme, was later developed by Ref. [28] to calculate time domain wave propagation analysis for boreholes in an anisotropic formation, using a fourth order discretization in space.

Tube waves were found to be a major source of noise, which made the data recorded at the receivers hard to interpret. Different data processing schemes have been suggested [29,30], but, in addition, more realistic models have been proposed to deal with the scattering of the tube waves caused by irregularities in the diameter of the borehole.

The effects of borehole irregularities on the Stoneleywave propagation have been addressed by Bouchon and Schmitt [31] using a boundary integral equation combining with a discrete wavenumber formulation. They concluded that the Stoneley wave propagation was not affected when the change in the borehole diameter along its axis was smooth, while a significant amount of reflection was registered when the diameter varied sharply. Later, Tezuka et al. [29] implemented a method for low-frequency Stoneleywave propagation in a borehole with both spatial variation of the borehole diameter along it axis and formation property changes. In this model, the main features of the lowfrequency Stoneley waves are maintained with a single 1-D model, while a mass-balance boundary condition and a propagator matrix are used to express Stoneley wave interactions with the borehole irregularities.

The remainder of this paper is concerned with the effect exercised by the existing non-circular fluid-filled boreholes, and computes the wavefield and motions elicited by monopole sources placed in off-center positions. Non-circular borehole cross-sections may occur for a variety of reasons, for example, through the mechanical action of the drill string in vertically deviated wells, rock failure adjacent to a drilled borehole, plastic deformation and washing out of the borehole in soft or poorly consolidated rocks $[32,33]$. Most of the published work has assumed that the borehole is circular and little work on non-circular fluid-filled boreholes has been reported. Randall [34] calculated dispersion curves for the modes of non-circular fluid-filled boreholes in homogeneous elastic formations, using a boundary integral formulation. Results for the propagation modes of several borehole shapes in both fast and slow formations were given. The boundary integral formulation used is similar to that used by Bouchon and Schmitt [31] to solve the problem of a circular, but axially varying borehole cross section. In the frequency versus axial wavenumber domain, the displacements and the stresses on the borehole are defined as integrals over surface distribution of effective sources. The unknown sources are calculated imposing the adequate boundary conditions, leading to a homogeneous linear system of equations. The zeros of the determinant of this system provide the modal dispersion functions. In his work, no frequency and time-domain waveforms are produced for any type of source excitation. In the present paper, we compute the wavefield and motions elicited by monopole sources placed in off-center positions, when the cross-section of the fluid-filled borehole is assumed to be irregular. Because of the cylindrical geometry of this problem, we can use the separation of variables and express the solution at each frequency in terms of waves with varying wavenumber, $k_{z}$, (with $z$ being the borehole axis), which we subsequently Fourier-transform into the spatial domain. We cast this wavenumber transform in discrete form by considering an infinite number of virtual point sources equally spaced along the $z$ axis, and at a sufficient distance from each other to avoid spatial contamination. In addition, our analysis uses complex frequencies, shifting the frequency axis downward-in the complex plane-in order to remove the singularities on (or near) the axis, and to minimize the influence of neighboring fictitious sources. The problem is solved using a BEM formulation containing compact expressions of Green's functions for the elastic and fluid media.

The article is organized as follows: first, a brief definition of the 3-D problem is given, and then the BEM is formulated in the frequency domain. Then follows a brief validation of the results using a circular cylindrical fluid-filled borehole model, for which analytical solutions are known. A circular fluid-filled borehole is also used in this work as a reference, to better understand the wave propagation changes when the cross-section of the borehole is non-circular. The theoretical part of the paper ends with a discussion of the dispersion of waves in a fluid-filled circular borehole. Finally, we give examples of simulations performed for a set of four crosssections. The solution is computed for a wide range of frequencies and wavenumbers, which then are used to obtain time series by means of (fast) inverse Fourier transforms into space-time. 


\section{Problem formulation}

Consider a cylindrical irregular cavity of infinite extent, buried in a spatially uniform elastic medium (Fig. 1), subjected to a harmonic dilatational point source at the position $O$, oscillating with a frequency $\omega$. The incident field can be expressed by means of the now classical dilatational potential $\phi$ :

$\phi_{\text {inc }}=\frac{A e^{i \frac{\omega}{\alpha}\left(\alpha t-\sqrt{x^{2}+y^{2}+z^{2}}\right)}}{\sqrt{x^{2}+y^{2}+z^{2}}}$

in which the subscript inc denotes the incident field, $A$ is the wave amplitude, $\alpha$ is the compressional wave velocity of the medium, and $i=\sqrt{-1}$.

Defining the effective wavenumbers:

$k_{\alpha}=\sqrt{\frac{\omega^{2}}{\alpha^{2}}-k_{z}^{2}}, \operatorname{Im} k_{\alpha}<0$

by means of the axial wavenumber $k_{z}$, and Fourier-transforming Eq. (1) in the $z$ direction, one obtains:

$\hat{\phi}_{\mathrm{inc}}\left(\omega, x, y, k_{z}\right)=\frac{-i A}{2} H_{0}^{(2)}\left(k_{\alpha} \sqrt{x^{2}+y^{2}}\right)$

in which the $H_{n}^{(2)}(\ldots)$ are second Hankel functions of order $n$.

Considering an infinite number of virtual point sources equally spaced along the $z$ direction, at a sufficient distance, $L$, from each other to avoid spatial contamination [35], the incident field may be written as:

$\phi_{\text {inc }}(\omega, x, y, z)=\frac{2 \pi}{L} \sum_{m=-\infty}^{\infty} \hat{\phi}_{\text {inc }}\left(\omega, x, y, k_{z}\right) e^{-i k_{z m} z}$

with:

$k_{z m}=\frac{2 \pi}{L} m$.

This equation converges and can be approximated by a finite sum of terms.

\section{Boundary element formulation}

The Boundary Element Method (BEM) is used to obtain the three-dimensional field generated by a cylindrical fluid filled borehole subjected to spatially sinusoidal harmonic line loads defined by Eq. (3). The fundamental equations underlying the application of boundary elements to wave propagation are well known [36-38]. It is, therefore, enough to state that the application of the method in the frequency domain requires, for the type of scattering problem presented here, the evaluation of the integrals

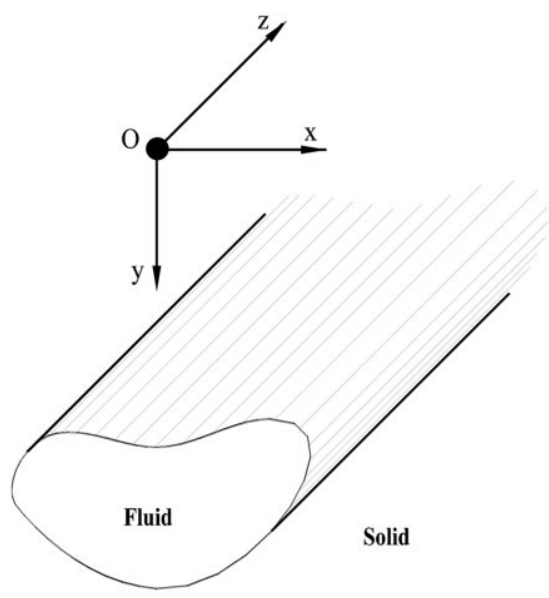

Fig. 1. Geometry of the problem.

along the appropriately discretized boundary of the borehole

$H_{i j}^{(s) k l}=\int_{C_{l}} H_{i j}^{(s)}\left(x_{k}, x_{l}, n_{l}\right) d C_{l} \quad(i, j=1,2,3)$

$H_{f 1}^{(f) k l}=\int_{C_{l}} H_{f 1}^{(f)}\left(x_{k}, x_{l}, n_{l}\right) d C_{l}$

$G_{i j}^{(s) k l}=\int_{C_{l}} G_{i j}^{(s)}\left(x_{k}, x_{l}\right) d C_{l} \quad(i=1,2,3 ; j=1)$

$G_{f 1}^{(f) k l}=\int_{C_{l}} G_{f 1}(f)\left(x_{k}, x_{l}\right) d C_{l}$

in which $H_{i j}^{(s)}\left(x_{k}, x_{l}, n_{l}\right)$ and $G_{i j}^{(s)}\left(x_{k}, x_{l}\right)$ are, respectively, the Green's tensor for traction and displacement components in the elastic medium, at the point $x_{l}$ in direction $j$ caused by a concentrated load acting at the source point $x_{k}$ in direction $i ; H_{f 1}^{(f)}\left(x_{k}, x_{l}, n_{l}\right)$ are the components of the Green's tensor for pressure in the fluid medium, at the point $x_{l}$ caused by a pressure load acting at the source point $x_{k} ; G_{f 1}^{(f)}\left(x_{k}, x_{l}\right)$ are the components of the Green's tensor for displacement in the fluid medium, at the point $x_{l}$ in the normal direction, caused by a pressure load acting at the source point $x_{k} ; n_{1}$ is the unit outward normal for the lst boundary segment $C_{1}$; the subscripts $i, j=1,2,3$ denote the normal, tangential and $z$ directions, respectively. These equations are conveniently transformed from the Cartesian coordinate system $(x, y, z)$ by means of standard vector transformation operators. The required two-and-a-half dimensional fundamental solution (Green's functions) in the Cartesian co-ordinate, for the elastic and fluid formations, are listed in Appendix A. Expressions for the tensions may be obtained from the Green's functions by taking partial derivatives to deduce the strains and then applying Hooke's law to obtain the stresses, as given in Appendix A.

After mathematical manipulation of the integral Eq. (5), which are combined and subjected to the continuity of normal displacements and stresses, and ascribing null tangential stresses at the interface between the solid and 


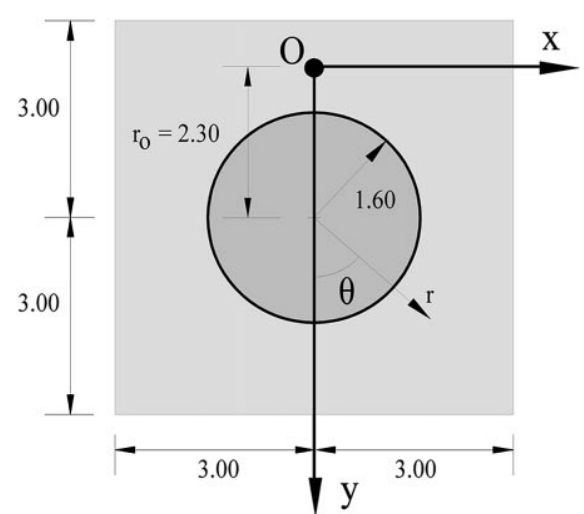

Fig. 2. Circular cylindrical inclusion in an unbounded elastic medium.

the fluid at the interface between the two media, we obtain a system of equations that can be solved for the nodal solid displacements and fluid pressures. The required integrations in Eq. (5) are performed using Gaussian quadrature when the element to be integrated is not the loaded element. For the loaded element, the existing singular integrands are carried out in closed form $[39,40]$.

\section{Validation of the BEM algorithm}

The BEM algorithm was implemented and validated by applying it to a cylindrical circular cavity filled with an inviscid fluid with a pressure wave velocity of $\alpha_{f}=1500 \mathrm{~m} /$ $\mathrm{s}$ and a density of $\rho_{f}=1000 \mathrm{~kg} / \mathrm{m}^{3}$, placed in a homogeneous elastic medium with a compressional wave velocity of $\alpha=4208 \mathrm{~m} / \mathrm{s}$, a shear wave velocity $\beta=2656 \mathrm{~m} / \mathrm{s}$ and a density of $\rho=2140 \mathrm{~kg} / \mathrm{m}^{3}$, subjected to a point dilatational load applied at point $O$, as in Fig. 2, for which the solution is known in closed form (described in Appendix B).

The response is calculated over a fine vertical grid plane, placed perpendicular to the longitudinal axis, as illustrated in Fig. 3. This figure also displays an example of a BEM mesh, including the nodal points in the middle of each constant boundary element. Fig. 3 represents the scattered pressure field inside the borehole and the $z$ displacement field in the solid formation, computed when a harmonic pressure load of $900 \mathrm{~Hz}$ is excited at $k_{z}=1.0 \mathrm{rad} / \mathrm{m}$. These figures give both the normalized response obtained with the closed form solution and the difference in the response obtained using the BEM (error), when the inclusion is modeled with a different number of boundary elements. Notice that the normalized response has been obtained by dividing the response by the maximum displacement and pressure, both outside and inside the borehole.

As expected, the BEM accuracy improves as shorter constant boundary elements are used to model the response. In the present example, the ratio between the wavelength of the shear waves and the length of boundary elements varies between six ( 20 boundary elements) and 12 (40 boundary elements). Analysis of the results also makes it possible to

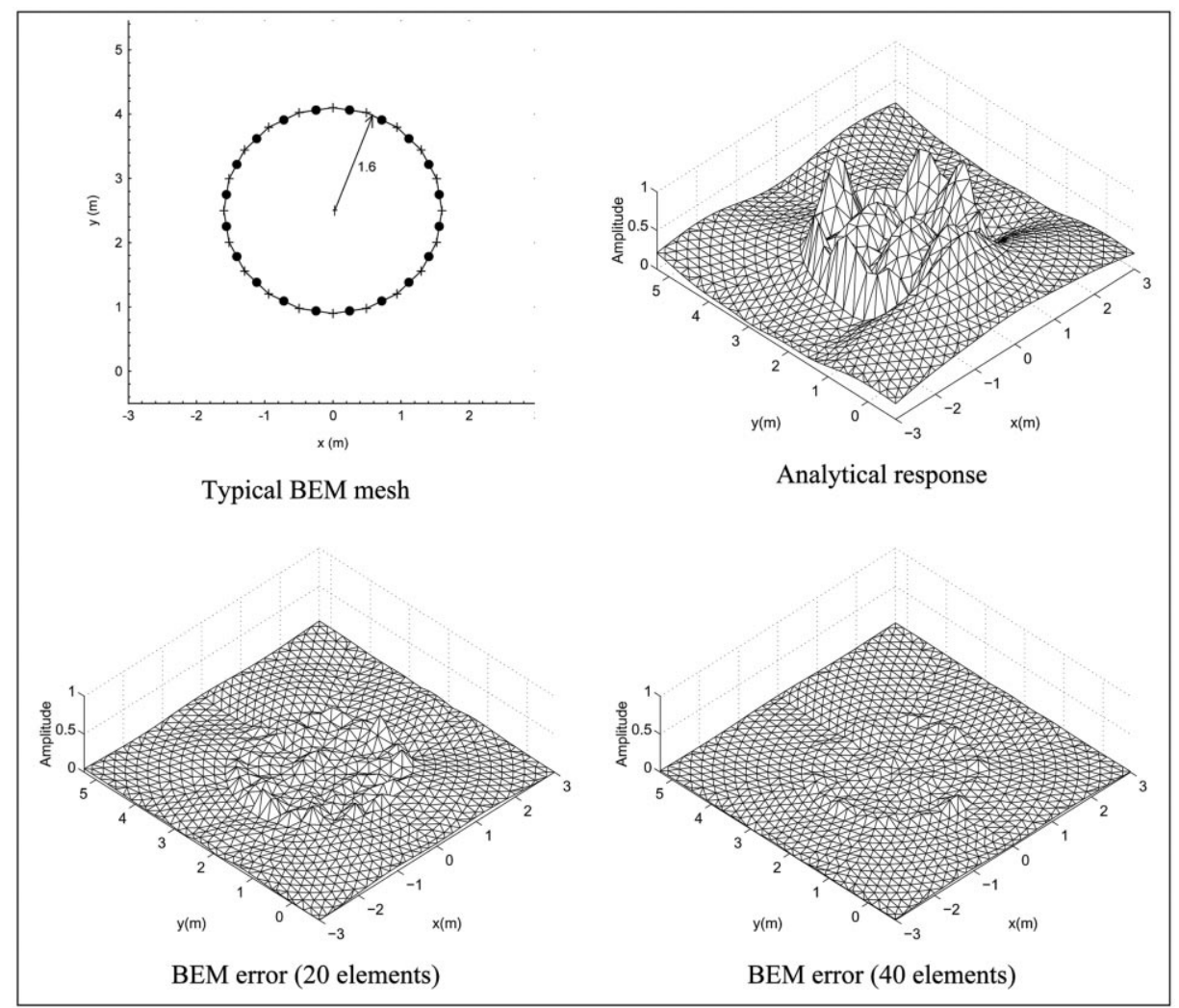

Fig. 3. Validation of the BEM. 
verify the improvement of the BEM solution as the distance to the surface of the inclusion increases.

\section{Results in the time domain}

The results (displacements and pressures) in the spatialtemporal domain are obtained by a numerical fast Fourier transform in $k_{z}$, considering a source whose temporal variation is given by a Ricker wavelet, as defined below. This wavelet form has been chosen because it decays rapidly, in both time and frequency; this not only reduces computational effort, but also allows easier interpretation of the computed time series and synthetic waveforms.

The Ricker wavelet function is given by:

$u(\tau)=A\left(1-2 \tau^{2}\right) e^{-\tau^{2}}$

where $A$ is the amplitude, $\tau=\left(t-t_{s}\right) / t_{0}$ and $t$ denotes time; $t_{s}$ is the time when the maximum occurs, while $\pi t_{0}$ is the characteristic (dominant) period of the wavelet. Its Fourier transform is:

$U(\omega)=A\left[2 \sqrt{\pi} t_{o} e^{-i \omega t_{s}}\right] \Omega^{2} e^{-\Omega^{2}}$

in which $\Omega=\omega t_{0} / 2$.

As stated before, the Fourier transformations are achieved by discrete summations over wavenumbers and frequencies, which is the mathematical equivalent of adding periodic sources at spatial intervals $L=2 \pi / \Delta k_{z}$ (in the $z$-axis), and temporal intervals $T=2 \pi / \Delta \omega$, with $\Delta k_{z}$, and $\Delta \omega$ being the wavenumber and frequency steps, respectively [35]. The spatial separation $L$ must be sufficiently large to avoid contamination of the response by the periodic sources. In other words, the contribution to the response by the fictitious sources must be guaranteed to occur at times later than $T$. Achievement of this goal can also be aided substantially by shifting the frequency axis slightly downward, that is, by considering complex frequencies with a small imaginary part of the form $\omega_{c}=\omega-i \eta$ (with $\eta=0.7 \Delta \omega$ ). This technique results in the significant attenuation, or virtual elimination, of the periodic sources. In the time domain, this shift is later taken into account by applying an exponential window $e^{\eta t}$ to the response [41].

\section{Dispersion of waves in a fluid-filled circular borehole}

Of the various waves propagating along the boundary of a fluid-filled cylinder, two are non-dispersive body waves, namely the dilatational $(P)$ and shear $(S)$ waves. When the source is within the fluid, the waves begin as dilatational waves in the fluid. As they reach the cylinder boundary, they are critically refracted into the formation as $P$ or $S$ waves, which are in turn refracted back into the fluid as $P$ waves. In addition, there are various types of guided waves-the normal modes-propagating along the interface between the fluid and solid. When the source is positioned on the axis of the cylinder, only the axisymmetric modes are excited. By contrast, additional modes with some azimuth variation are excited when the source is placed away from the axis, but these modes do not contribute to the pressures recorded at receivers positioned on the axis. Certain modes, however, are excited only if the source excitation frequency exceeds the cut-off (or resonant) frequencies of the cylinder.

The dispersion characteristics of the normal modes can be obtained by solving an eigenvalue problem in the absence of an incident field. The associated eigenvalues $k_{z}$, lead in turn to the phase and group velocities of the normal modes. While an infinite (but countable) number of modes exists, only those with low modal order contribute significantly to the response.

The normal modes depend strongly on the ratio $\beta / \alpha_{f}$ between the shear wave velocity in the solid medium and the dilatational wave velocity in the fluid. If this ratio is less than one (i.e., a slow formation), proper normal modes do not exist because any waves propagating in the fluid will radiate and lose their energy as shear waves in the solid. However, in this case one can still find modes with a complex wavenumber (referred to as leaky modes), which attenuate rapidly as they propagate. On the other hand, if the stated ratio is larger than one (a fast formation), energy may remain trapped in the fluid cylinder and normal modes do exist; in addition, there may also be leaky modes. In general, the amplitude of the normal modes in the formation decays exponentially with distance to the cylinder. Thus, the phase and group velocities of all normal modes must be smaller than the shear wave velocity in the formation, except of course for the leaky modes.

Next, we consider a fluid filled borehole (radius $=0.6 \mathrm{~m}$ ) placed inside two different formations, studied by Ref. [42]: Fast formation

$\alpha=4208 \mathrm{~m} / \mathrm{s}$

$\beta=2656 \mathrm{~m} / \mathrm{s}$

$\rho=2140 \mathrm{~kg} / \mathrm{m}^{3}$

$\alpha_{f}=1500 \mathrm{~m} / \mathrm{s}$

$\rho_{f}=1000 \mathrm{~kg} / \mathrm{m}^{3}$

Slow formation

$\alpha=2630 \mathrm{~m} / \mathrm{s}$

$\beta=1416 \mathrm{~m} / \mathrm{s}$

$\rho=2250 \mathrm{~kg} / \mathrm{m}^{3}$

$\alpha_{f}=1500 \mathrm{~m} / \mathrm{s}$

$\rho_{f}=1000 \mathrm{~kg} / \mathrm{m}^{3}$

\subsection{Fast formation}

Fig. 4 gives the phase and group velocities for the lowest order of normal modes in the fast formation. In these figures, the modes are identified by a pair of numbers. The first number of the pair is the azimuth order, which indicates the variation of the mode with the azimuth, while the second is the radial order that supplies the variation of the mode with radial distance. The first axisymmetric mode is the tube wave or Stoneley wave. The tube wave exists for all frequencies, and is 

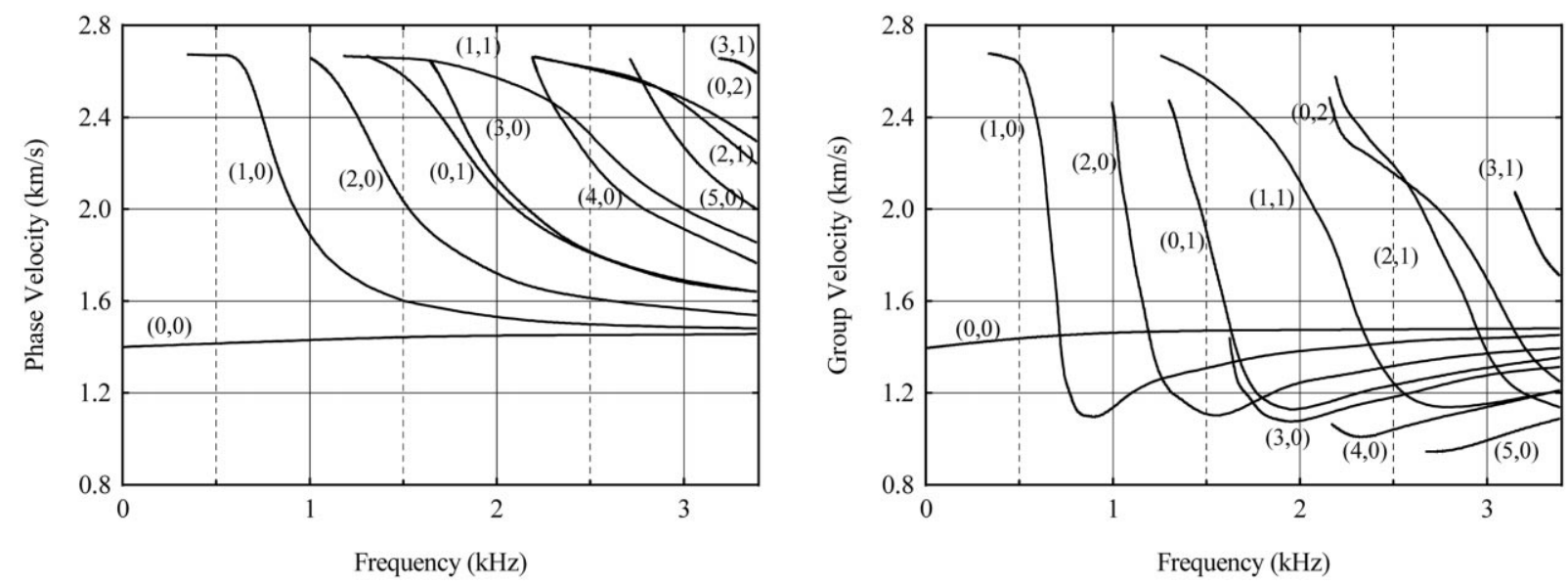

Fig. 4. Fast formation. Phase and group velocities of the lowest normal modes.

associated, in Fig. 4, with the pair $[(0,0)]$ as shown. This wave exhibits a slight dispersion and its energy is usually highest at low frequencies. Its phase and group velocity values are below the dilatational velocity of the fluid.

The second axisymmetric mode is the Pseudo-Rayleigh wave $[(0,1)]$, which, in this case has a cut-off frequency of $1.35 \mathrm{kHz}$. At this cut-off frequency, the mode reaches its highest phase velocity, namely the shear wave velocity of the solid. As the frequency of the wave increases, the phase velocity of the mode approaches asymptotically, from above the fluid velocity. The group velocity value, however, is less than the shear wave velocity of the solid at a low-frequency cut-off. From this point on, it decreases rapidly with frequency, reaching the minimum associated with Airy waves, and thereafter approaches from below the fluid velocity. The PseudoRayleigh wave is thus highly dispersive.

There is also an infinite number of higher order PseudoRaleigh waves $[(0,2),(0,3), \ldots]$ which have correspondingly higher cut-off frequencies. Within the fluid cylinder, these waves are oscillatory in nature and decay with distance from the axis. The amplitude of the first mode, for example, has a zero value at a point located at (approxi-

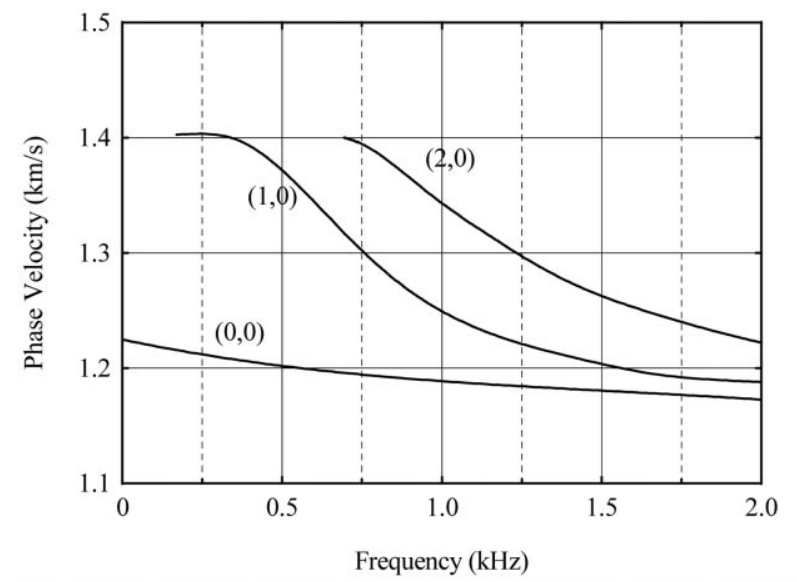

mately) $2 / 3$ of the distance between the axis and the borehole wall.

The first mode with azimuth variation is the Flexural wave $[(1,0)]$, which exists at all frequencies. The fluid pressure for this mode varies as the cosine or sine of the azimuth angle. As can be seen in Fig. 4, this mode is highly dispersive. Higher Flexural modes also exist, such as the $[(1,1)]$ mode shown.

The second azimuth modes, which vary as $\cos 2 \theta$ or $\sin 2 \theta$, are referred to as the Screw waves. The fundamental Screw wave $[(2,0)]$ has a cut-off frequency of approximately $1 \mathrm{kHz}$. Again, an infinite number of higher order modes exist $[(2,1),(2,2) \ldots]$.

Higher order normal modes $[(n, m)]$ for $m, n>1$ which vary as $\cos n \theta$ or $\sin n \theta$ also exist, but these do not have a common name.

\subsection{Slow formation}

The phase and group velocities for the lowest normal modes, calculated for the slow formation, are presented in Fig. 5. When the shear wave velocity is lower than the

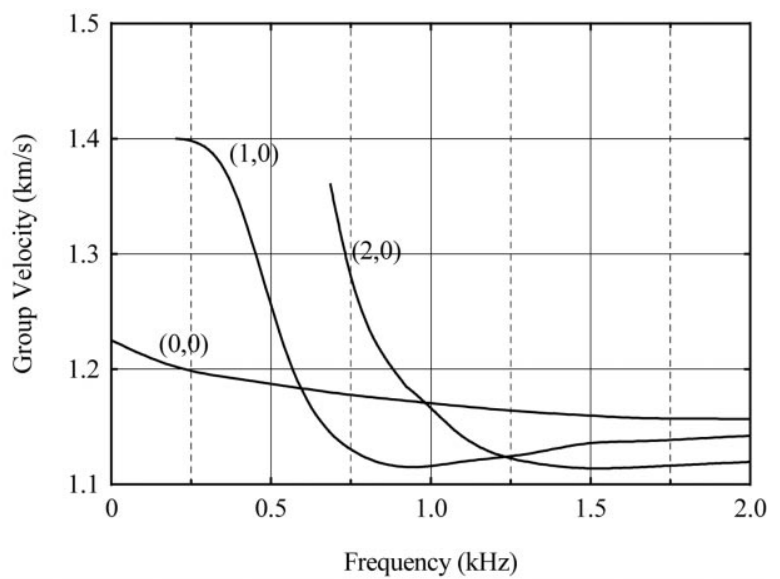

Fig. 5. Slow formation. Phase and group velocities of the lowest normal modes. 

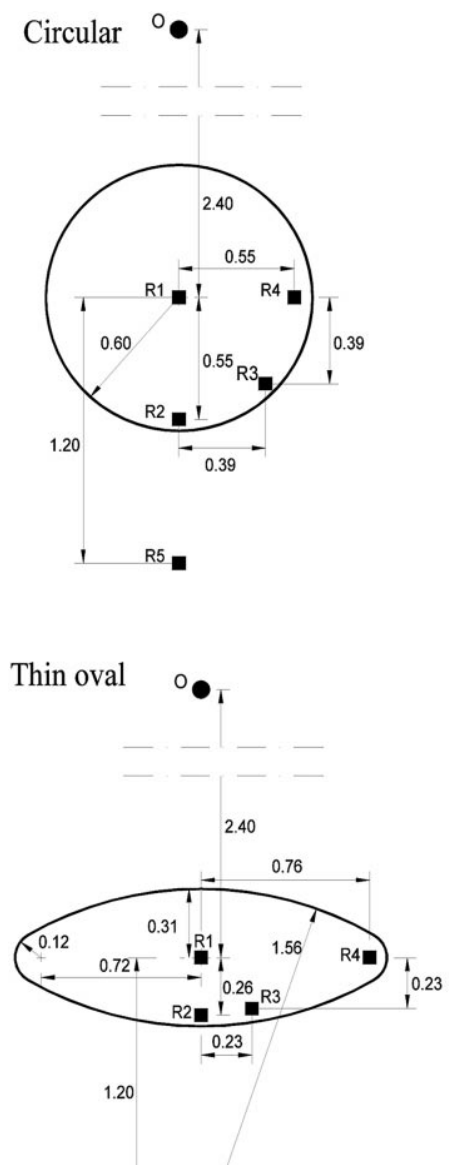
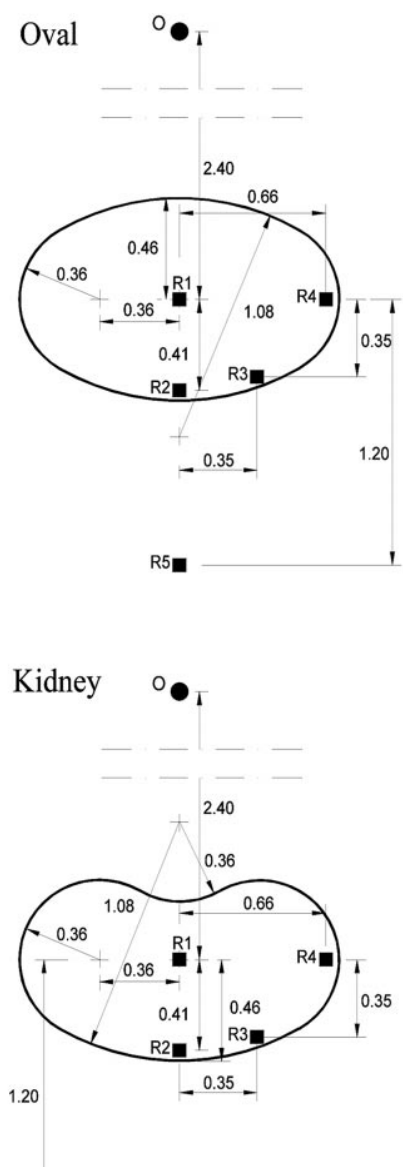

Fig. 6. Cross-section of the fluid-filled boreholes.

dilatational fluid velocity, only the lowest order radial modes exist $[(0,0),(1,0),(2.0) \ldots]$. Thus, the only surviving axisymmetric mode is the tube wave (Stoneley wave). The Pseudo-Rayleigh waves no longer exist since no critical shear refraction can occur. The Stoneley wave is more dispersive in a slow formation than in a fast formation. Relations for the Flexural and the Screw modes are also shown in Fig. 5. The cut-off frequency of the Screw wave is approximately $0.7 \mathrm{kHz}$, whereas the Flexural wave is always propagating. Both waves are less dispersive in slow than in fast formations.

\section{Synthetic waveforms}

Next we present the wave signatures at a set of receivers placed inside and outside boreholes, with four different cross-sections: circular; oval with an ovality ratio of $\epsilon=1.44 / 0.92=1.56$; a thin oval with an ovality ratio of $\epsilon=1.68 / 0.62=2.7$, and kidney-shaped. The perimeter of all these boreholes is constant $(1.2 \pi m)$, as shown in Fig. 6, which displays the geometry of the cross-sections. At time $t=0$, a point source at a point $O$ creates a spherical dilatational pulse propagating away from $O$.

The inclusions are modeled with boundary elements, the number of which changes with the frequency of excitation of the harmonic load. A ratio of the wavelength of the incident waves to length of the boundary elements is kept at a minimum of 6.0. In all cases the minimum number of the boundary elements used is less than 24 .

The field generated is computed at receivers, located on five planes, equally spaced $(6 \mathrm{~m})$ along the $z$ direction. The geometry of the plane containing the point source is illustrated in Fig. 6.

\subsection{Fast formation}

We performed our computations in the frequency range from $12.5 \mathrm{~Hz}$ to $1600 \mathrm{~Hz}$, with a frequency increment of $12.5 \mathrm{~Hz}$; thus, the total time taken for the analysis is $T=1 / 12.5=80 \mathrm{~ms}$. This implies, in turn, that the spatial period cannot be less than $L=2 T \alpha_{s}=673 \mathrm{~m}$. We chose $\eta=0.7 \Delta \omega$ as the imaginary part of the angular frequency, to attenuate the wraparound by a factor of $e^{0.7 \Delta \omega T}=81$ (i.e., $38 \mathrm{~dB}$ ). The source is a Ricker wavelet pulse with a characteristic frequency of $500 \mathrm{~Hz}$. In all cases, the computed wave fields refer to pressure when the receiver is in the fluid, and to vertical ( $y$ ) displacements when the receiver is in the solid formation. Also, within each time plot the different 


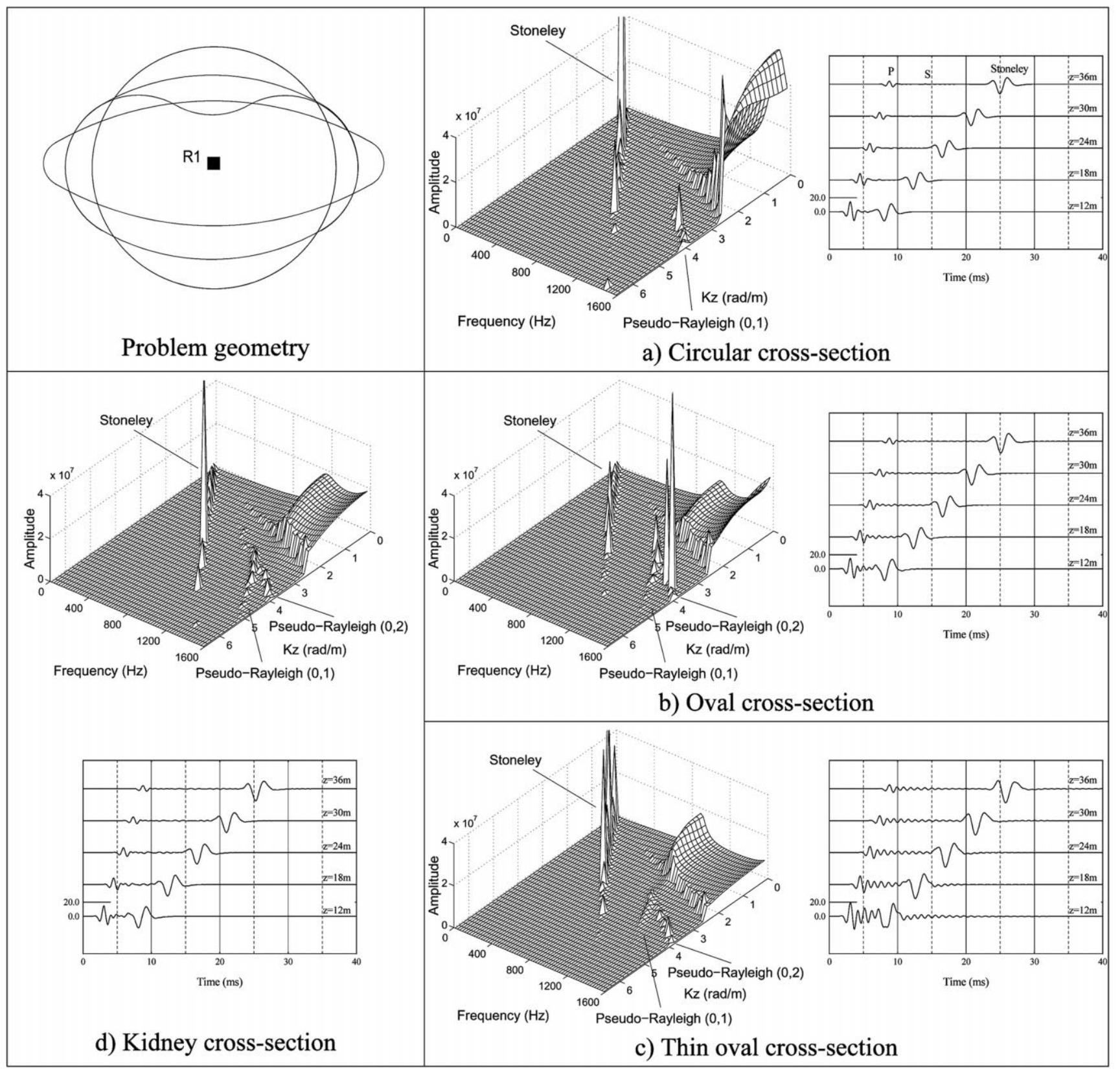

Fig. 7. Responses at receivers R1 (Fast formation).

responses at the various receivers placed along the $z$ axis keep the same scale.

Fig. 7 displays the response at receiver 1, placed on the axis, obtained for the four different cross-sections of fluidfilled inclusions analyzed in this work. Both the time responses and their Fourier Spectra representations are included, for a better visual separation of the different wave types. Indeed, the amplitude of the wavefield in the frequency vs axial-wave number domain allows an easier recognition, identification, and physical interpretation of the different wave components.

A source placed off the axis excites both axisymmetric and non-axisymmetric modes, but only the former contri- bute to the pressure on the axis if the inclusion is regular. Hence, in the case of the circular, oval and thin oval formations, only the body waves, the Stoneley waves $[(0,0)]$, and the Pseudo-Rayleigh waves $[(0,1)]$ are observed. The response there is similar to what would be generated by a source on the axis (not shown), except that the amplitudes of the response are now lower, and the importance of the Stoneley wave greater than for centered sources.

The response calculated for the circular inclusion follows the above-defined phase and group velocity curves. Indeed, the time arrivals of the different pulses agree with the velocities of the $P, S$, and Stoneley waves defined in these curves. The time plots do not show 


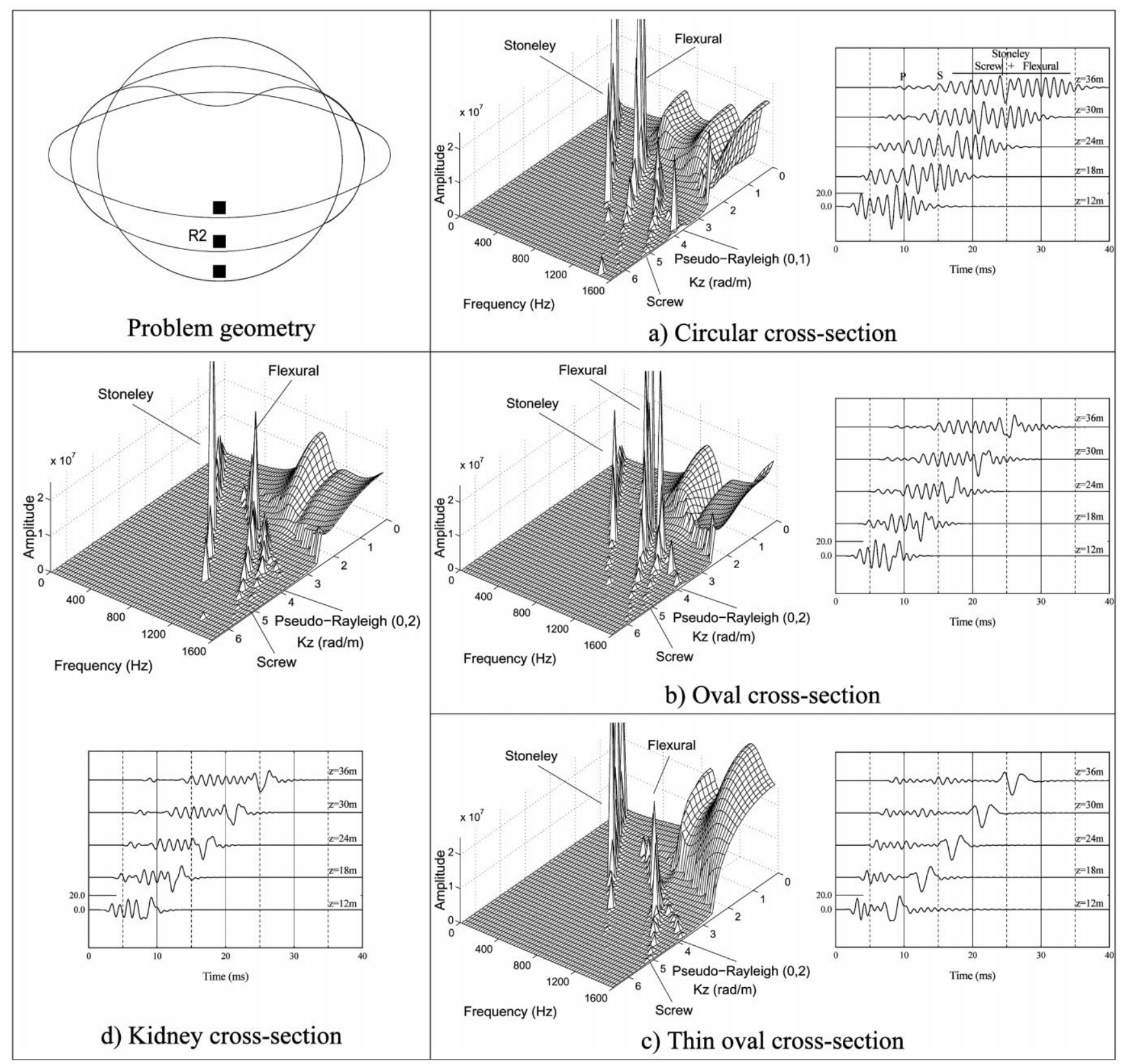

Fig. 8. Responses at receivers R2 (Fast formation).

evidence of the existence of the Pseudo-Rayleigh pulses because of the low frequency characteristic of the excited Ricker pulse $(500 \mathrm{~Hz})$, which is much lower than the cutoff frequency of these waves $(1.35 \mathrm{kHz})$. As we move from the circular to the oval $\epsilon=1.56$, and then to the thin oval $\epsilon=2.7$, the spectra plots exhibit a progressively slower tube wave. The time plots confirm this phenomenon by placing the Stoneley pulses with a progressively delayed arrival. This effect agrees with the tendency found by Ref. [34] in the calculation of the dispersion curves for modes of non-circular fluid-filled boreholes for low frequencies. Additionally, as we move from the circular to the oval, and then to the thin oval-shaped inclusions, our results indicate that the amplitude of the Stoneley pulses increases, and the arrival of a growing ring packed with $P$ waves can be seen. The oscillatory tail corresponds to leaky modes generated by the interaction of the formation with totally reflected $P$ waves in the borehole. Similar behavior would occur for a circular inclusion with a smaller radius or a higher source frequency excitation. Furthermore, it is observed that the Pseudo-Rayleigh cut-off frequency decreases as the ovality ratio increases. A second higher order Pseudo-Rayleigh $[(0,2)]$ can even be observed. The time responses reflect the presence of these waves, exhibiting a ring of pulses after the $S$ wave arrives, which decreases rapidly, given the low frequency 


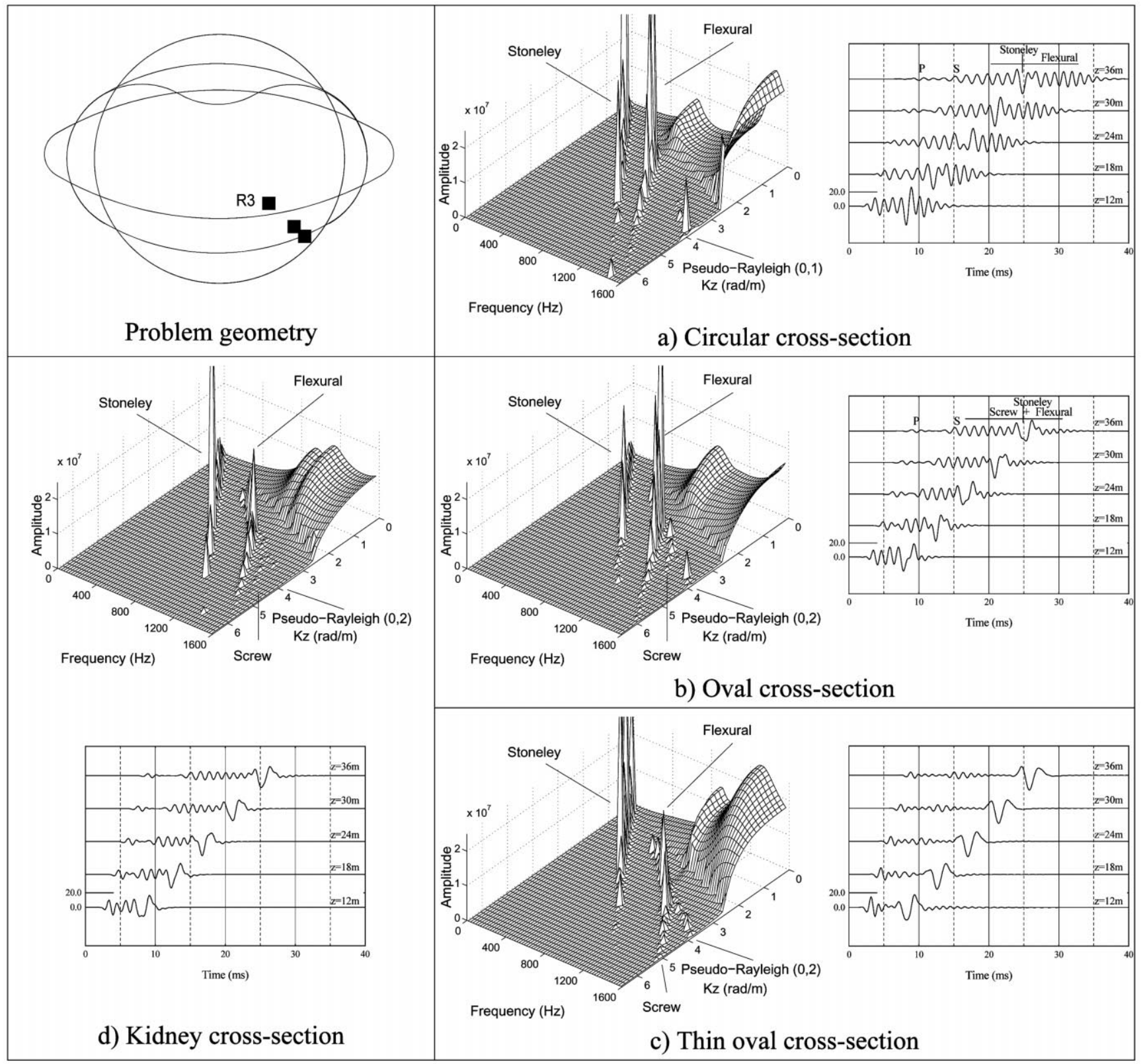

Fig. 9. Responses at receivers R3 (Fast formation).

of the pulse excited. The importance of these waves decreases as the ovality ratio increases.

The responses obtained for the kidney shaped inclusion are closely related to the ones calculated for the oval $(\epsilon=1.56)$. It seems that the wall deformation of the oval does not cause a significant modification of the former axisymmetric modes behavior.

Fig. 8 illustrates the response close to the wall of the borehole, at receiver 2, placed in the same azimuth direction of the source. The responses at this receiver include contributions from the Flexural and Screw waves in addition to the body, Stoneley and Pseudo-Rayleigh waves.

Analysis of the responses shows that the amplitude of the $P$ wave registered a stronger value on the axis (recei- ver 1) for all four cases, the result of the accumulation of $P$ waves emitted from points located on the periphery of the borehole. The Stoneley wave is visible and its amplitude is higher than the amplitude of the $P$ wave, but slightly bigger than its amplitude on the axis (receiver 1), as would be expected. It also retains the same behavior found at receiver 1 , when we move from the circular to the other inclusions. The Pseudo-Rayleigh waves lose their importance, as may be seen on the spectral representation of the response.

As we have mentioned, the non-axisymmetric guided waves for the circular inclusion mainly arise from the first order Flexural waves $[(1,0)]$ since the Screw waves $[(2,0)]$ have a cut-off frequency of approximately $1 \mathrm{kHz}$. 


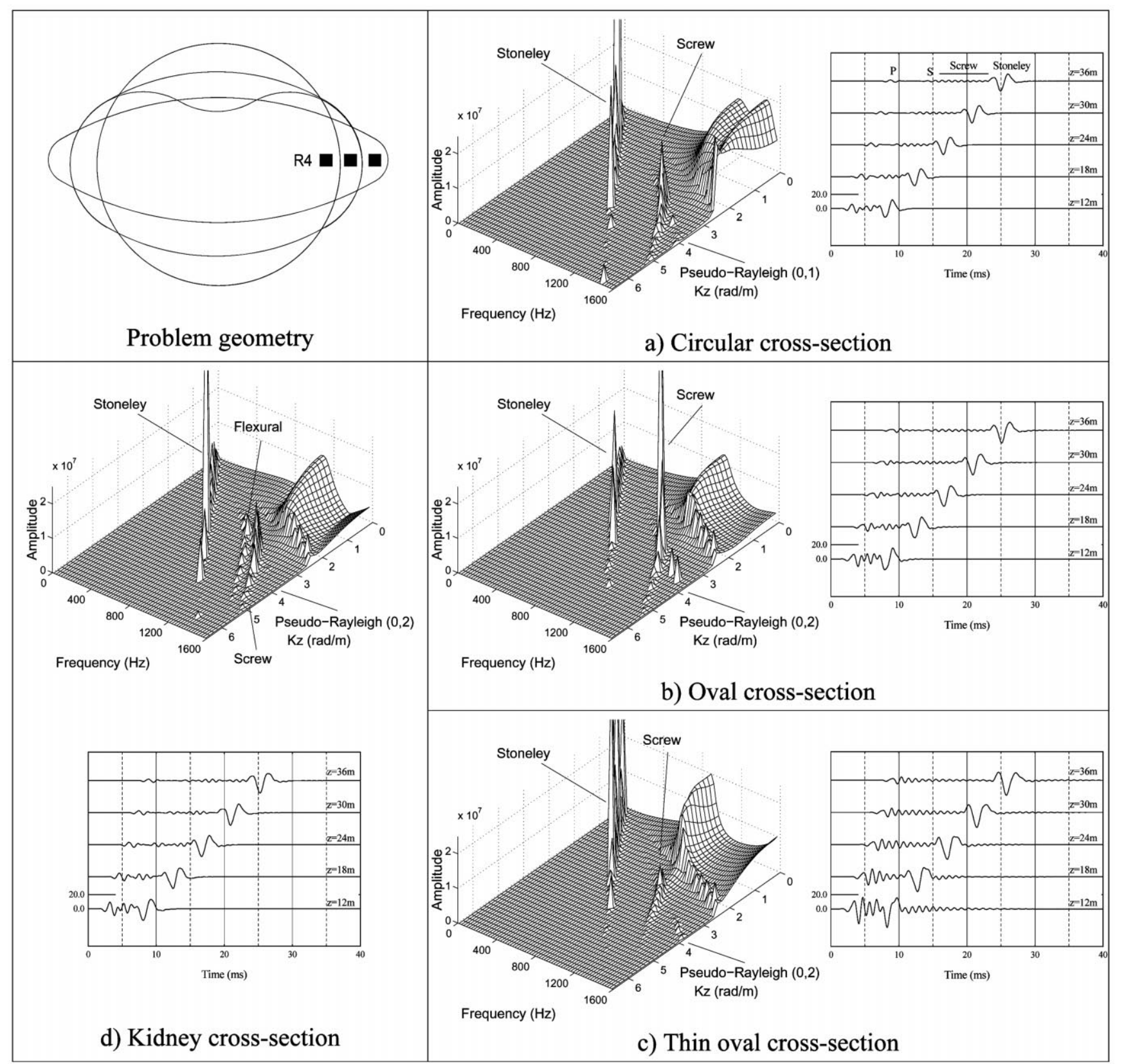

Fig. 10. Responses at receivers R4 (Fast formation).

If we consider the spectral representation of the response (Fig. 8(a)), we can visually separate the different wave types. It indicates that the group velocity of bending waves is lower than that of Screw waves, which in turn is lower than for Pseudo-Rayleigh waves. As we move from the circular inclusion to the oval, and then to the thin oval, we notice that the guided waves lose their importance (Fig. 8(a)-(c)). Furthermore, it can be observed that the Flexural waves reach higher velocities at lower frequencies, approaching the velocity of the Flexural waves found for the circular inclusion, for later frequencies, as the ovality ratio increases. In the time plots this can be seen in the progressive disappearance of late arrivals, after the Stone- ley wave. The Screw wave behaves in a similar way, but, in addition, it rapidly loses importance.

The responses obtained for the kidney cross-section inclusion are again closely related to those calculated for the oval $(\epsilon=1.56)$, but with a pronounced decay in the amplitude of the Screw and Flexural waves. It seems that, in this case, the oval wall deformation causes a sharp modification in the non-axisymmetric modes' behavior.

We can also observe that the signatures at receiver 4 are not affected by Flexural waves (Fig. 10), when the inclusion is regular, because the bending mode has zero amplitude on the neutral axis, which is the horizontal plane through the axis that is perpendicular to the line connecting the center with the 


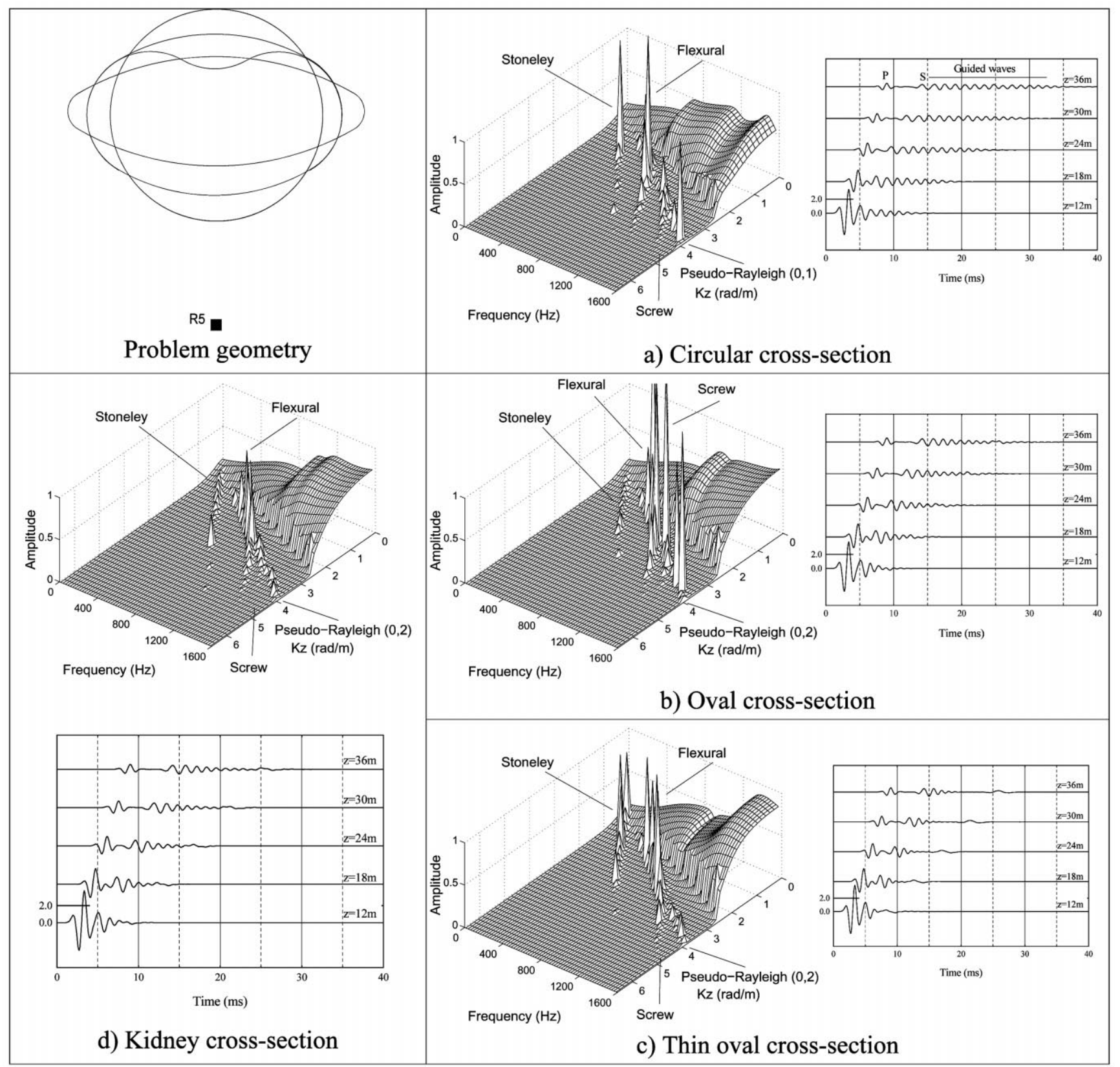

Fig. 11. Responses at receivers R5 (Fast formation).

source. Hence, after the passage of the Stoneley wave, the signatures on the neutral axis experience a substantial drop in amplitude (Fig. 10(a)-(c)). This drop, however, is not observed for receivers 2 , and 3 located away from the neutral axis (Figs. 8 and 9). Indeed, receivers 2 and 3 that are farthest from the neutral axis exhibit the largest increases in pressure, which is clearly due to the Flexural mode. As mentioned, at receiver 4 , the only surviving guided waves, when the borehole has a regular cross-section, are those arising from the Screw mode. We may now compare the results calculated at receiver 1 with those at receiver 4 , and conclude once again, that the Screw mode loses its influence as the ovality ratio increases from $\epsilon=1.56$ to $\epsilon=2.7$.
The kidney cross-section inclusion produces results showing the presence of the Flexural wave (Fig. 10(d)). This behavior was expected since receiver 4 is not on the neutral axis of the inclusion, because of its side wall deformation. However, its influence is very slight, as we may conclude by comparing these responses with those given by the oval borehole.

Trailing behind the Flexural waves is a set of pulses associated with the Screw mode. We can see that their contribution to the pressure at receiver 3 , which lies on a $45^{\circ}$ plane, is very small, as theory would predict for the circular cavity. It can be seen that the Screw mode is not null on this $45^{\circ}$ plane when the cross-section of the inclusion is non-circular. 


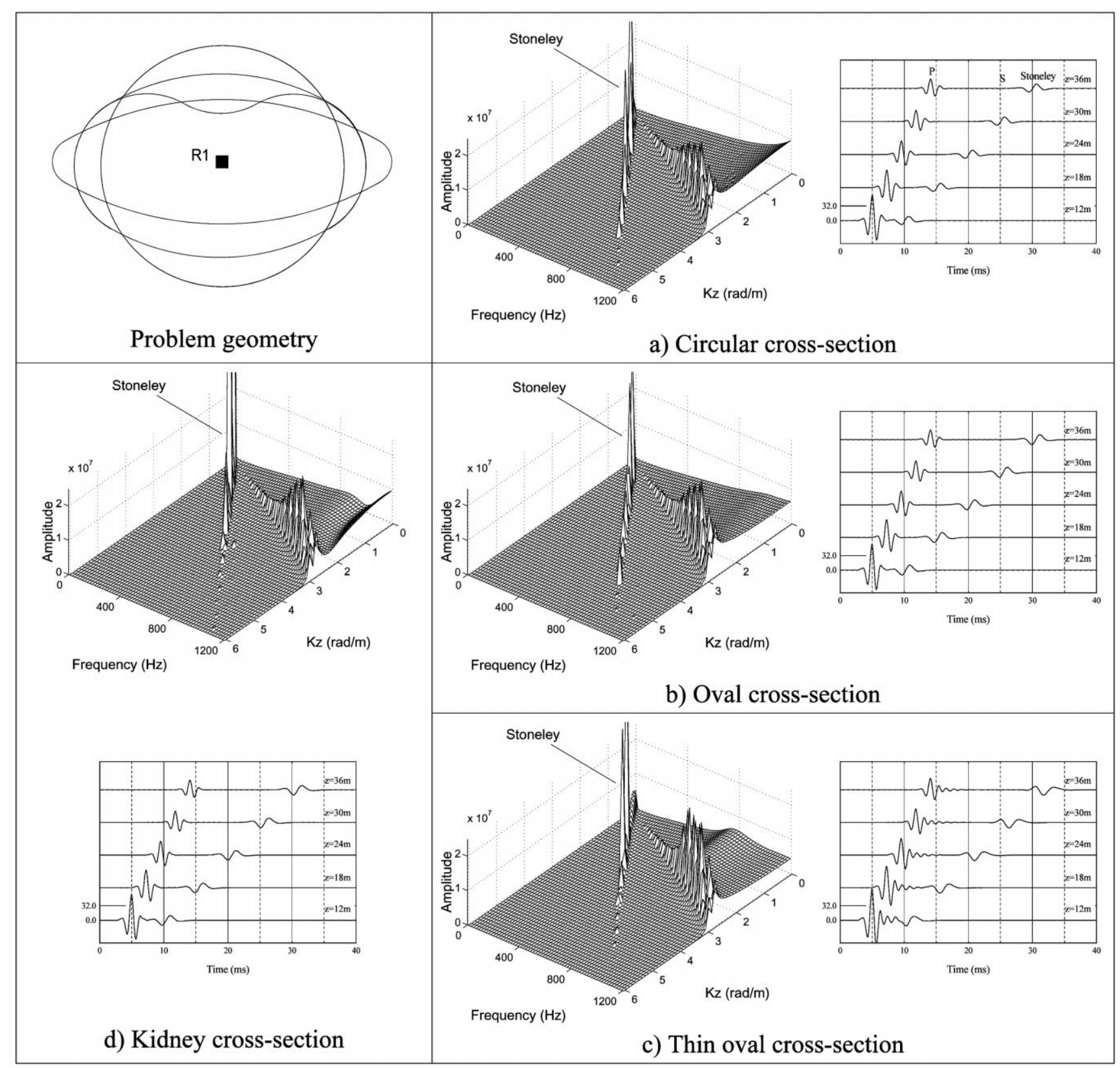

Fig. 12. Responses at receivers R1 (Slow formation).

However, its influence on the time response is very small, given its cut-off frequency.

The response for receiver 5 (Fig. 11), placed outside the borehole, shows some of the features generated at receiver 2 . The guided waves undergo significant decay, but their contribution is still important. Again, the Stoneley pulse increases its importance, while the non-axisymmetric guided waves lose their importance, as we move from the circular to the oval, and then to the thin oval-shaped borehole. Furthermore, it can be observed that the Flexural waves reach higher velocities at lower frequencies, approaching the shear velocity, before slowing down as the frequency increases. The time plots show this in the progressive disappearance of late arrivals, after the Shear wave. The Screw wave follows similar behavior, but it again rapidly loses importance.

\subsection{Slow formation}

The computations are performed in the frequency range $(9.5 \mathrm{~Hz}, 1216 \mathrm{~Hz})$, with a frequency increment of $9.5 \mathrm{~Hz}$, which determines the total time duration $(T=105.2 \mathrm{~ms})$ for the analysis in the time domain. The source time dependence is a Ricker wavelet with a characteristic frequency of $450 \mathrm{~Hz}$; $\eta=0.7 \Delta \omega$ is again the imaginary part of the angular frequency. The computed wave fields refer to pressure for 


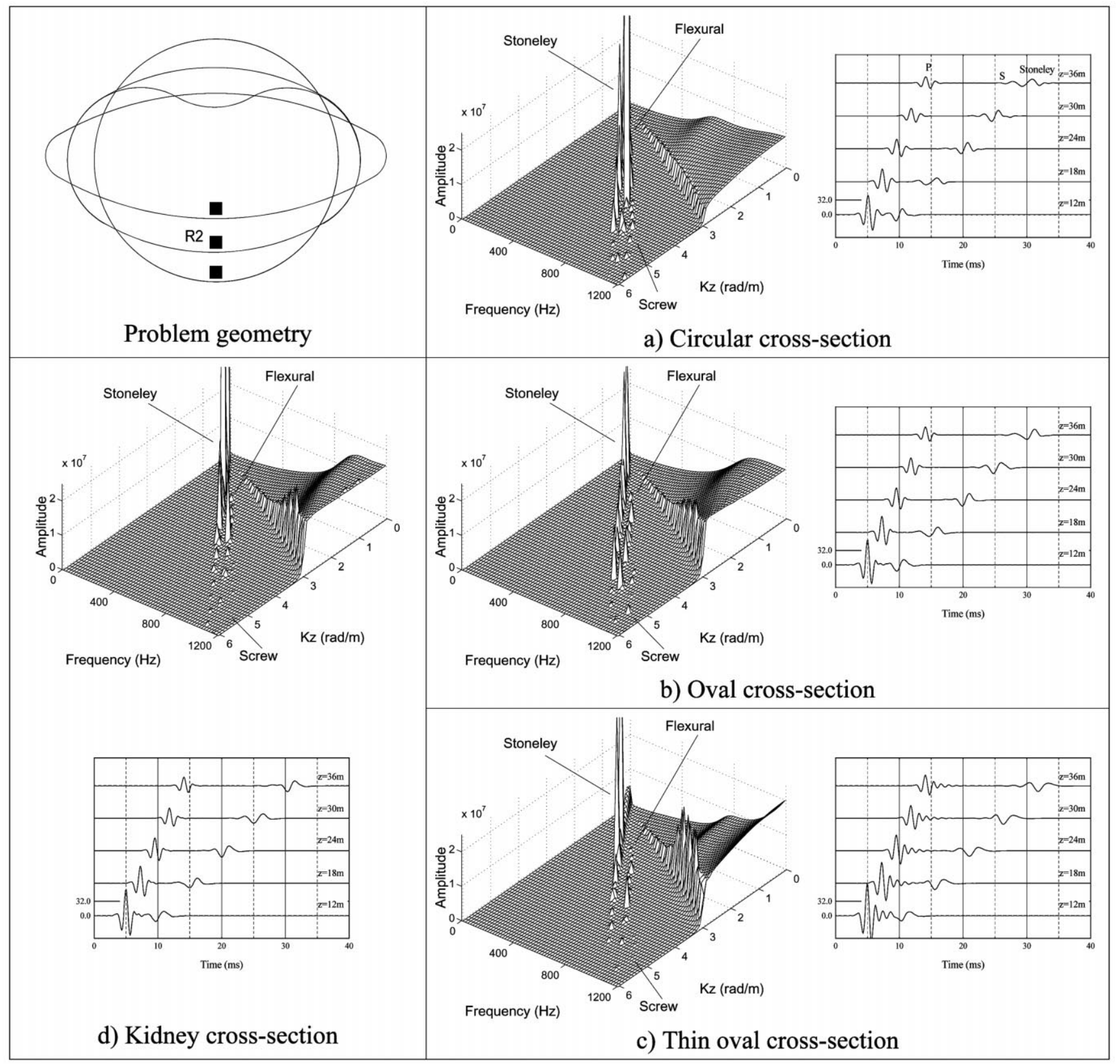

Fig. 13. Responses at receivers R2 (Slow formation).

receivers placed within the borehole, and to vertical (y) displacements when the receiver is in the solid medium.

Fig. 12 shows the calculated responses when the receiver is at position 1 , on the axis. The $P$ arrival is very pronounced and its character is distinctly different from that in the fast formation. Once more, as we move from the circular inclusion to the oval and then to the thin oval, an oscillatory $P$ tail appears, which corresponds to the leaky modes generated by the interaction of the formation with totally reflected $P$ waves in the borehole. Again, the Stoneley wave manifests a slower velocity as the ovality ratio increases, as observed from the time plots. At receiver 2 (Fig. 13), it is possible to identify the presence of the Flexural wave, but its relative contribution is smaller than before (note that Flexural waves propagate for all frequencies). The participation of the Screw waves is very small because its cut-off frequency is approximately $0.7 \mathrm{kHz}$. The features of the guided waves in these plots, when we move from the circular inclusion to the others, are similar to those for the fast formation. Only the results obtained for receiver 2 are presented, as an example.

\section{Conclusions}

The boundary element formulation developed and 
implemented to evaluate the 3-D scattered field generated by a dilatational point load illuminating non-circular fluidfilled boreholes was found to be efficient. It was therefore used to assess the influence of receiver position on the propagation of both axisymmetric and non-axisymmetric wave modes when different borehole cross-sections were used, namely a circular, an oval, a thin oval and a kidney. Both the time responses and their Fourier Spectral representations were included to give better identification of the different wave components.

As we move from the circular to the oval cross-section borehole, placed in a fast formation, the results indicate a progressively slower tube wave with an increasing amplitude and the arrival of an expanding ring packed of $P$ waves as the ovality ratio increases. The oscillatory tail corresponds to leaky modes generated by the interaction of the formation with totally reflected $P$ waves in the borehole. Similar behavior would be found for a circular inclusion with a smaller radius, or for a higher source frequency excitation. Furthermore, it is also observed that the Pseudo-Rayleigh cut-off frequency decreases as the ovality ratio increases. In some cases, the appearance of a second higher order Pseudo-Rayleigh $[(0,2)]$ can even be observed. The responses obtained when the wall of an oval-shaped inclusion is deformed (designated here as the kidney shape inclusion), are closely related to the ones calculated for the former oval, when the receiver is in its center.

The non-axisymmetric guided waves were mainly caused by the first order Flexural waves $[(1,0)]$ since the Screw waves $[(2,0)]$ have a high cut-off frequency. As we moved from the circular inclusion to the oval, we noticed that the guided waves lost their importance. Furthermore, it was found that the Flexural waves reached higher velocities at lower frequencies, approaching the velocity of the Flexural waves registered for the circular inclusion for later frequencies, as the ovality ratio increases. The Screw waves behave similarly, but they also rapidly lose importance. The responses obtained for the kidney crosssection inclusion are again closely related to those found for the oval one, but with a pronounced decay in the amplitude of the Screw wave.

The response at a receiver placed outside the borehole showed some of the features found at a receiver placed within the fluid medium, close to the borehole wall. The guided waves undergo significant decay, but their contribution is still important. Again, the Stoneley pulse increases in importance, while the non-axisymmetric guided wave loses its importance, as we move from the circular to the oval inclusion.

In a slow formation, the $P$ arrivals were very pronounced and their character is quite different from that found in the fast formation. Again as we moved from the circular to the oval inclusion, an oscillatory $P$ tail appeared again, corresponding to the leaky modes generated by the interaction of the formation with totally reflected $P$ waves in the borehole. As for the fast formation, the Stoneley wave showed a slower velocity as the ovality ratio increased. The features of the guided waves for non-circular inclusions are similar to those for the fast formation, its importance, however, decreases.

\section{Appendix A. The Green's functions}

\section{A.1. Solid formation}

Definitions:

$$
\begin{array}{ll}
\lambda, \mu & \text { Lamé constants } \\
\rho & \text { Mass density } \\
\alpha=\sqrt{(\lambda+2 \mu) / \rho} & P \text { wave velocity } \\
\beta=\sqrt{\mu / \rho} & S \text { wave velocity } \\
k_{p}=\omega / \alpha, & k_{s}=\omega / \beta \\
k_{\alpha}=\sqrt{k_{p}^{2}-k_{z}^{2}} & k_{\beta}=\sqrt{k_{s}^{2}-k_{z}^{2}} \\
A=\frac{1}{4 i \rho \omega^{2}} & \text { Amplitude } \\
\gamma_{i}=\partial r / \partial x_{i}=x_{i} / r, \quad i=1,2 & \text { Direction cosines } \\
H_{n \alpha}=H_{n}^{(2)}\left(k_{\alpha} r\right), \quad H_{n \beta}=H_{n}^{(2)}\left(k_{\beta} r\right) & \text { Hankel functions } \\
B_{n}=k_{\beta}^{n} H_{n \beta}-k_{\alpha}^{n} H_{n \alpha} & B_{n} \text { functions }
\end{array}
$$

Green's functions for displacements:

$$
\begin{aligned}
& G_{x x}=A\left[k_{s}^{2} H_{0 \beta}-\frac{1}{r} B_{1}+\gamma_{x}^{2} B_{2}\right] \\
& G_{y y}=A\left[k_{s}^{2} H_{0 \beta}-\frac{1}{r} B_{1}+\gamma_{y}^{2} B_{2}\right] \\
& G_{z z}=A\left[k_{s}^{2} H_{0 \beta}-k_{z}^{2} B_{0}\right] \\
& G_{x y}=G_{y x}=\gamma_{x} \gamma_{y} A B_{2} \\
& G_{x z}=G_{z x}=i k_{z} \gamma_{x} A B_{1} \\
& G_{y z}=G_{z y}=i k_{z} \gamma_{y} A B_{1}
\end{aligned}
$$

Volumetric strain (super-index $=$ direction of load):

$$
\begin{aligned}
\epsilon_{\mathrm{Vol}}^{l} & =G_{x l, j}+G_{y l, l}+G_{z l, z} \\
& =A\left[\frac{\partial}{\partial x_{l}}\left(k_{s}^{2} H_{0 \beta}\right)+B_{0, x l x}+B_{0, y l y}+B_{0, z l z}\right] \\
& =A \frac{\partial}{\partial x_{l}}\left[k_{s}^{2} H_{0 \beta}+B_{0, x x}+B_{0, y y}+B_{0, z z}\right] \\
& =A \frac{\partial}{\partial x_{l}}\left[k_{s}^{2} H_{0 \beta}+\hat{\nabla}^{2} B_{0}\right]
\end{aligned}
$$

Note:

$$
H_{0 \beta, l}=-k_{\beta} \gamma_{l} H_{l \beta} \quad H_{0 \beta, z}=-i k_{z} H_{0 \beta}
$$


Strain components (tensor definition, not engineering):

$$
\begin{aligned}
\epsilon_{i j}^{l} & =\frac{1}{2}\left(G_{i l, j}+G_{j l, i}\right)=\frac{1}{2} A\left(\delta_{i l} k_{s}^{2} H_{0 \beta, j}+\delta_{j l} k_{s}^{2} H_{0 \beta, i}+B_{0, i l j}+B_{0, j l i}\right) \\
& =\frac{1}{2} k_{s}^{2} A\left(\delta_{i l} H_{0 \beta, j}+\delta_{j l} H_{0 \beta, i}\right)+A B_{0, i j l}
\end{aligned}
$$

1. Strains for loads in the plane, $1=x, y$

$$
\begin{aligned}
& \epsilon_{\mathrm{Vol}}^{l}=\gamma_{l} A\left(-k_{s}^{2} k_{\beta} H_{1 \beta}+k_{z}^{2} B_{1}+\frac{4}{r} B_{2}-B_{3}\right) \\
& \epsilon_{x x}^{l}=\gamma_{l} A\left(\left(\frac{2}{r} B_{2}-k_{s}^{2} k_{\beta} H_{1 \beta}\right) \delta_{x l}+\frac{1}{r} B_{2}-\gamma_{x}^{2} B_{3}\right)(\mathrm{A} 10) \\
& \epsilon_{y y}^{l}=\gamma_{l} A\left(\left(\frac{2}{r} B_{2}-k_{s}^{2} k_{\beta} H_{1 \beta}\right) \delta_{y l}+\frac{1}{r} B_{2}-\gamma_{y}^{2} B_{3}\right)(\mathrm{A} 11) \\
& \epsilon_{z z}^{l}=\gamma_{l} k_{z}^{2} A B_{1} \\
& \epsilon_{x y}^{l}=A\left(\left(\frac{1}{r} B_{2}-\frac{1}{2} k_{s}^{2} k_{\beta} H_{1 \beta}\right)\left(\delta_{x l} \gamma_{y}+\delta_{y l} \gamma_{x}\right)-\gamma_{x} \gamma_{y} \gamma_{l} B_{3}\right) \\
& \epsilon_{x z}^{l}=i k_{z} A\left(\left(\frac{1}{r} B_{1}-\frac{1}{2} k_{s}^{2} H_{0 \beta}\right) \delta_{x l}-\gamma_{x} \gamma_{l} B_{2}\right) \\
& \epsilon_{y z}^{l}=i k_{z} A\left(\left(\frac{1}{r} B_{1}-\frac{1}{2} k_{s}^{2} H_{0 \beta}\right) \delta_{y l}-\gamma_{y} \gamma_{l} B_{2}\right)
\end{aligned}
$$

2. Strain for axial loads, $1=z$ :

$$
\begin{aligned}
& \epsilon_{\mathrm{Vol}}^{z}=i k_{z} A\left(-k_{s}^{2} H_{0 \beta}+k_{z}^{2} B_{0}+\frac{2}{r} B_{1}-B_{2}\right) \\
& \epsilon_{x x}^{z}=i k_{z} A\left(\frac{1}{r} B_{1}-\gamma_{x}^{2} B_{2}\right) \\
& \epsilon_{y y}^{z}=i k_{z} A\left(\frac{1}{r} B_{1}-\gamma_{y}^{2} B_{2}\right) \\
& \epsilon_{z z}^{z}=i k_{z} A\left(-k_{s}^{2} H_{0 \beta}+k_{z}^{2} B_{0}\right) \\
& \epsilon_{x y}^{z}=-i k_{z} \gamma_{x} \gamma_{y} A B_{2} \\
& \epsilon_{x z}^{z}=\gamma_{x} A\left(-\frac{1}{2} k_{s}^{2} k_{\beta} H_{1 \beta}+k_{z}^{2} B_{1}\right) \\
& \epsilon_{y z}^{z}=\gamma_{y} A\left(-\frac{1}{2} k_{s}^{2} k_{\beta} H_{1 \beta}+k_{z}^{2} B_{1}\right)
\end{aligned}
$$

Stresses:

$$
\tau_{i j}^{l}=\lambda \epsilon_{V o l}^{l} \delta_{i j}+2 \mu \epsilon_{i j}^{l}
$$

\section{A.2. Fluid formation}

Definitions:

$$
\begin{aligned}
& \lambda_{f} \\
& \rho_{f} \\
& \alpha_{f} \\
& k_{p f}=\omega / \alpha_{f} \\
& k_{\alpha f}=\sqrt{k_{p f}^{2}-k_{z}^{2}} \\
& A_{f}=1 / 4 i \\
& \gamma_{i}=\partial r / \partial x_{i}=x_{i} / r i=1,2 \\
& H_{n \alpha f}=H_{n}^{(2)}\left(k_{\alpha f} r\right)
\end{aligned}
$$

Lamé constant

Mass density

$P$ wave velocity
Green's functions for displacements:

$G_{f x}=-A_{f} k_{\alpha f} H_{1 \alpha f} \gamma_{x}$

$G_{f y}=-A_{f} k_{\alpha f} H_{1 \alpha f} \gamma_{y}$

Stresses:

$H_{f 1}=A_{f} \lambda_{f} H_{0 \alpha f}\left(-\omega^{2} / \alpha_{f}^{2}\right)$

\section{Appendix B. 3-D wave propagation solution for an analytical fluid-filled circular borehole}

Consider a spatially uniform elastic medium of infinite extent, having a cylindrical cavity filled with an inviscid fluid (Fig. 2). Decomposing the homogeneous wave equations for elastic media in the usual way, by means of the now classical dilatational potential $\phi$ and shear potentials $\Psi, \chi$, one arrives at the three scalar wave equations in these potentials, with associated wave propagation velocities $\alpha$, and $\beta$, respectively. For a harmonic dilatational point source at an off-center position $O$ in the fluid or solid, oscillating with a frequency $\omega$, the scalar wave equations lead to three Helmholtz equations, whose solution can be expressed in terms of the single dilatational potential for the incident waves, together with the set of potentials for scattered waves in both media.

\section{B.1. Incident field (or free-field)}

The incident dilatational potential is given by the expression:

$\phi_{\text {inc }}=\frac{A e^{i \frac{\omega}{\alpha}\left(\alpha t-\sqrt{x^{2}+y^{2}+z^{2}}\right)}}{\sqrt{x^{2}+y^{2}+z^{2}}}$

in which the subscript inc denotes the incident field, $A$ is the wave amplitude, $\alpha$ is the acoustic (dilatational) wave velocity of the medium containing the source, and $i=\sqrt{-1}$. 
Defining the effective wavenumbers:

$k_{\alpha}=\sqrt{\frac{\omega^{2}}{\alpha^{2}}-k_{z}^{2}}, \operatorname{Im} k_{\alpha}<0$,

$k_{\beta}=\sqrt{\frac{\omega^{2}}{\beta^{2}}-k_{z}^{2}}, \quad \operatorname{Im} k_{\alpha}<0$

by means of the axial wavenumber $k_{z}$, the frequency of excitation $\omega$, and the wave velocities $\alpha, \beta$, and Fouriertransforming Eq. (B1) in the $z$ direction, one obtains:

$\phi_{\text {inc }}\left(\omega, x, y, k_{z}\right)=\frac{-i A}{2} H_{0}^{(2)}\left(k_{\alpha} \sqrt{x^{2}+y^{2}}\right)$

in which the $H_{n}^{(2)}(\ldots)$ are second Hankel functions of order $n$.

Eq. (3) poses a difficulty, however, because it expresses the incident field in terms of waves centered at the source point $O$, and not at the axis of the borehole. This difficulty can be overcome by expressing the incident potential in terms of waves centered at the origin, which can be achieved by resorting to Graf's addition theorem [43], leading to the expressions (in cylindrical coordinates):

$\phi_{\text {inc }}\left(\omega, r, \theta, k_{z}\right)=-\frac{i A}{2} \sum_{n=0}^{\infty}(-1)^{n} \epsilon_{n} H_{n}^{(2)}\left(k_{\alpha} r_{0}\right) J_{n}\left(k_{\alpha} r\right) \cos n \theta$

when $r<r_{0}$

$\phi_{\text {inc }}\left(\omega, r, \theta, k_{z}\right)=-\frac{i A}{2} \sum_{n=0}^{\infty}(-1)^{n} \epsilon_{n} H_{n}^{(2)}\left(k_{\alpha} r\right) J_{n}\left(k_{\alpha} r_{0}\right) \cos n \theta$

when $r>r_{0}$

in which the $J_{n}^{(2)}(\ldots)$ are Bessel functions of order $n, \theta$ is the azimuth, and:

$\epsilon_{n}=\left\{\begin{array}{lll}\frac{1}{2} & \text { if } & n=0 \\ 1 & \text { if } & n \neq 0\end{array}\right.$

$r=\sqrt{x^{2}+y^{2}}=$ radial distance to the receiver

$r_{0} \quad=$ radial distance from the cylinder axis to the source

$\cos \theta=x / r, \quad \sin \theta=y / r$

\section{B.2. Scattered field in the exterior region (solid)}

In the frequency-axial-wavenumber domain, the scattered field in the exterior region (the solid formation) can be expressed in a form similar to that of the incident field, namely

$\phi_{\mathrm{sca}}^{\mathrm{s}}\left(\omega, r, \theta, k_{z}\right)=\sum_{n=0}^{\infty} A_{n} H_{n}^{(2)}\left(k_{\alpha} r\right) \cos n \theta$

$\psi_{\mathrm{sca}}^{s}\left(\omega, r, \theta, k_{z}\right)=\sum_{n=0}^{\infty} B_{n} H_{n}^{(2)}\left(k_{\beta} r\right) \sin n \theta$ $\chi_{\text {sca }}^{s}\left(\omega, r, \theta, k_{z}\right)=\sum_{n=0}^{\infty} C_{n} H_{n}^{(2)}\left(k_{\beta} r\right) \cos n \theta$

in which the subscript sca denotes the scattered field, $A_{n}, B_{n}$, and $C_{n}$, are as yet unknown coefficients to be determined from appropriate boundary conditions. Together with an implicit factor expi $\left(\omega t-k_{z} z\right)$, the second Hankel functions in Eq. (B6) represent diverging or outgoing cylindrical waves.

\section{B.3. Scattered field in the interior region (fluid)}

If the source is outside the borehole (i.e., within the solid formation), the scattered (or refracted) field in the fluid consists of standing waves, which can be expressed as:

$\phi_{\mathrm{sca}}^{f}\left(\omega, r, \theta, k_{z}\right)=\sum_{n=0}^{\infty} D_{n} J_{n}\left(k_{\alpha f} r\right) \cos n \theta$

where the index $f$ identifying the fluid, and:

$k_{\alpha f}=\sqrt{\frac{\omega^{2}}{\alpha_{f}^{2}}-k_{z}}$

\section{B.4. Displacement field}

The unknown constants $A_{n}, B_{n}, C_{n}, D_{n}$ in Eqs. (B6 and B7) are obtained by imposing the continuity of displacements and stresses at the interface between the solid and the fluid, namely $u_{r}=u_{r}^{f}, \sigma_{r r}=\sigma_{r r}^{f}$, and $\sigma_{r \theta}=\sigma_{r z}=0$. Notice that since we have assumed an inviscid fluid, the tangential displacements at the boundary of the solid (i.e., $u_{\theta}, u_{z}$ ) may be different from those in the fluid (i.e., $u_{\theta}^{f}, u_{z}^{f}$ ). The imposition of the four stated boundary conditions for each summation index, $n$, leads to a system of four equations in the four unknown constants.

Having obtained the constants, we may compute the motions associated with the scattered field by means of the well-known equations relating potentials and displacements. In essence, this requires the application of partial derivatives of Eq. (B6) as well as Eq. (B7), to move from potentials to displacements. After carrying out this procedure, one obtains expressions for the scattered field in the solid and fluid of the form:

$u_{r}\left(\omega, r, \theta, k_{z}\right)=\sum_{n=0}^{\infty} f_{n}(r) \cos n \theta$

$u_{\theta}\left(\omega, r, \theta, k_{z}\right)=\sum_{n=0}^{\infty} g_{n}(r) \sin n \theta$

$u_{z}\left(\omega, r, \theta, k_{z}\right)=\sum_{n=0}^{\infty} h_{n}(r) \cos n \theta$ 
in which the functions $f_{n}, g_{n}$ and $h_{n}$ are given by:

Solid

$$
\begin{aligned}
f_{n}(r)= & {\left[\frac{n}{r} H_{n}^{(2)}\left(k_{\alpha} r\right)-k_{\alpha} H_{n+1}^{(2)}\left(k_{\alpha} r\right)\right] A_{n}+\frac{n}{r} H_{n}^{(2)}\left(k_{\beta} r\right) B_{n} } \\
& -i k_{z}\left[\frac{n}{r} H_{n}^{(2)}\left(k_{\beta} r\right)-k_{\beta} H_{n+1}^{(2)}\left(k_{\beta} r\right)\right] C_{n}
\end{aligned}
$$

$$
\begin{aligned}
g_{n}(r)= & -\frac{n}{r} H_{n}^{(2)}\left(k_{\alpha} r\right) A_{n}-\left[\frac{n}{r} H_{n}^{(2)}\left(k_{\beta} r\right)-k_{\beta} H_{n+1}^{(2)} k_{\beta} r\right] B_{n} \\
& +i k_{z} \frac{n}{r} H_{n}^{(2)}\left(k_{\beta} r\right) C_{n}
\end{aligned}
$$$$
h_{n}(r)=-i k_{z} H_{n}^{(2)}\left(k_{\alpha} r\right) A_{n}+k_{\beta}^{2} H_{n}^{2)}\left(k_{\beta} r\right) C_{n}
$$

Fluid

$$
\begin{aligned}
& f_{n}(r)=\left[\frac{n}{r} J_{n}\left(k_{\alpha f} r\right)-k_{\alpha f} J_{n+1}\left(k_{\alpha f} r\right)\right] D_{n} \\
& g_{n}(r)=-\frac{n}{r} J_{n}\left(k_{\alpha f} r\right) D_{n} \\
& h_{n}(r)=-i k_{z} J_{n}\left(k_{\alpha f} r\right) D_{n}
\end{aligned}
$$

From these equations, it follows that on the axis of the borehole, only the $n=0$ terms survive, and we retrieve the expressions derived earlier by Ref. [5]. On the other hand, at points away from the axis, the displacements are also a function of the non-axisymmetric terms with $n>0$. Finally, the incident field obtained from Eqs. (B4) or (B5) by partial differentiation must be added to Eq. (B9), for the medium containing the source, to give the total field in the $k_{z}$, wavenumber domain.

\section{References}

[1] Pao YH. Diffraction of elastic waves and dynamic stress concentrations. Rand Corporation, Santa Monica, CA, USA, 1973.

[2] Biot MA. Propagation of elastic waves in cylindrical bore containing a fluid. J Appl Phys 1952;23:997-1005.

[3] Blair DP. Rise times of attenuated seismic pulses detected in both empty and fluid-filled cylindrical boreholes. Geophysics 1984;49:398-410.

[4] Schoenberg M. Fluid and solid motion in the neighborhood of a fluidfilled borehole due to the passage of a low frequency elastic plane wave. Geophysics 1986;51:1191-205.

[5] Lovell JR, Hornby BE. Borehole coupling at sonic frequencies. Geophysics 1990;55:806-14.

[6] Roever WL, Rosenbaum JH, Vining TF. Acoustic waves from an impulsive source in a fluid-filled borehole. J Acoust Soc Am 1974;55:1144-57.

[7] Tsang L, Rader D. Numerical evaluation of the transient acoustic waveform due to a point source in a fluid-filled borehole. Geophysics 1979;44:1706-20.

[8] Cheng CH, Toksöz MN. Elastic wave propagation in a fluid-filled borehole and synthetic acoustic logs. Geophysics 1981;46:104253.

[9] Kurkjian AL. Numerical computation of individual far-field arrivals excited by an acoustic source in a borehole. Geophysics 1985;50:852-6.
[10] Kurkjian AL, Chang S-K. Acoustic multipole sources in fluid-filled boreholes. Geophysics 1986;51:148-63.

[11] Siggins AF, Stokes AN. Circumferential propagation of elastic waves on boreholes and cylindrical cavities. Geophysics 1987;52:514-29.

[12] Cheng CH, Zhang J, Burns DR. Effects of in-situ permeability on the propagation of Stoneley (tube) waves in a borehole. Geophysics 1987;52:1279-89.

[13] Paillet FL, White JE. Acoustic modes of propagation in the borehole and their relationship to rock properties. Geophysics 1982;47:1215-28.

[14] Baker LJ. The effect of the invaded zone on full wavetrain acoustic logging. Geophysics 1984;49:796-809.

[15] Schmitt DP, Bouchon M. Full-wave acoustic logging: synthetic microseis-mograms and frequency-wavenumber analysis. Geophysics 1985;50:1756-78.

[16] Baker LJ, Winbow GA. Multipole $P$-wave logging in formations altered by drilling. Geophysics 1988;53:1207-18.

[17] White JE, Tongtaow C. Cylindrical waves in transversely isotropic media. J Acoust Soc Am 1981;70:1147-55.

[18] Chan AK, Tsang L. Propagation of acoustic waves in a fluid-filled borehole surrounded by a concentrically layered transversely isotropic formation. J Acoust Soc Am 1983;74:1605-16.

[19] Schmitt DP. Acoustic multipole logging in transversely isotropic poroelastic formations. J Acoust Soc Am 1989;86:2397-421.

[20] Ellefsen KJ. Elastic wave propagation along a borehole in an anisotropic medium, PhD thesis. Cambridge, MA: MIT, 1990.

[21] Sinha BK, Norris AN, Chang SK. Borehole flexural modes in anisotropic formations. 61st S.E.G Annual Meeting Expanded Abstracts, Houston, 1991.

[22] Norris AN, Sinha BK. Weak elastic anisotropy and the tube wave. Geophysics 1993;58:1091-8.

[23] Dong W, Toksöz NM. Borehole seismic-source radiation pattern in transversely isotropic media. Geophysics 1995;60:29-42.

[24] Pratt RG, Chapman CH. Traveltime tomography in anisotropic media: application. Geophys J Internat 1992;109:20-37.

[25] Kurkjian AL, Coates RT, White JE, Schmidt H. Finite-difference and frequency-wavenumber modeling of seismic monopole sources and receivers in fluid-filled boreholes. Geophysics 1994;59:1053-64.

[26] Peng C, Lee JM, Toksöz NM. Pressure in a fluid-filled borehole caused by a seismic source in stratified media. Geophysics 1996;61:43-55.

[27] Randall CT. Multipole acoustic waveforms in nonaxisymmetric boreholes and formations. J Acoust Soc Am 1991;90:1620-31.

[28] Cheng N, Cheng CH, Toksöz MN. Borehole wave propagation in three dimensions. J Acoust Soc Am 1995;97:3483-93.

[29] Tezuka K, Cheng CH, Tang XM. Modeling of low-frequency Stoneley-wave propagation in an irregular borehole. Geophysics 1997;62:1047-58.

[30] Herman GC, Milligan PA, Dong Q. Analysis and removal of multiply scattered tube waves. Geophysics 2000;65:745-54.

[31] Bouchon M, Schmitt DP. Full wave acoustic logging in an irregular borehole. Geophysics 1989;54:758-65.

[32] Bell JS, Gough DI. Northeast-southwest compressive stress in Alberta-evidence from oil wells. Earth Planet Sci Lett 1979;45:47582.

[33] Zheng Z, Kemeny K, Cook NGW. Analysis of borehole breakouts. J Geophy Res 1989;94:171-182.

[34] Randall CT. Modes of noncircular fluid-filled boreholes in elastic formation. J Acoust Soc Am 1991;89:1002-16.

[35] Bouchon M, Aki Keiti. Discrete wave-number representation of seismic source wavefields. Bull Seism Soc Am 1977;67:259-77.

[36] Manolis GD, Beskos DE. Boundary element methods in elastodynamics. London: Unwin Hyman, 1988 (sold to Chapman and Hall).

[37] Beskos DE. Boundary element methods in dynamic analysis: Part II (1986-1996). Appl Mech Rev 1997;50(3):149-97.

[38] Brebbia CA, Telles JCF, Wrobel LC. Boundary element techniques. Wessex Institute of Technology, WIT Press, UK, 1984.

[39] Tadeu AJB, Santos PFA, Kausel E. Closed-form integration of 
singular terms for constant, linear and quadratic boundary elementspart I: SH wave propagation. EABE-Engineering Analysis with Boundary Elements 1999;23(8):671-81.

[40] Tadeu AJB, Santos PFA, Kausel E. Closed-form integration of singular terms for constant, linear and quadratic boundary elements-part II: SV-P wave propagation. EABE-Engineering Analysis with Boundary Elements 1999;23(9):757-68.
[41] Kausel E. Frequency domain analysis of undamped systems. Journal of Engineering Mechanics, ASCE 1992;118:121-134.

[42] Ellefsen KJ. Elastic wave propagation along a borehole in an anisotropic medium, PhD. Department of Earth and Planetary Sciences, MIT, MASS, USA, 1990

[43] Watson GN. A treatise on the theory of Bessel functions. 2nd edition. Cambridge University Press, Cambridge, UK, 1980. 